\title{
THE EFFECT OF SOME SOIL TREATMENT AND FOLIAR APPLICATIONS ON THE PRODUCTIVITY AND QUALITY OF PEPPER CROP \\ 1-VEGETATIVE GROWTH, YIELD AND CHEMICAL \\ Hala A. El-Sayed ${ }^{*}$; S. M. Farid ${ }^{\star *}$ and Rania E. El-Zehery ${ }^{\star *}$ \\ *Veg. and Flori. Dept., Fac. of Agric; Mans. Univ.,Egypt. \\ ${ }^{*}$ Veg. Res. Dept., Hort. Res. Ins., Agric. Res. Center, Giza, Egypt.
}

\begin{abstract}
Two field experiments were conducted on bell pepper (Capsicum annuumL.) variety of " California wonder" plants during the summer seasons of 2012 and 2013 at EL-Baramon experimental farm near EL-Mansoura, Dakahlia Governorate Egypt to study the effect of -planting treatments as well as their interactions on growth and productivity of bell pepper. Fifteen treatments were arranged in split plots design, which were the simple possible combination between three treatments of soil application (Straw rice costs (4 ton/fed.), Humic acid (5 kg/fed.), and Polymers (20 $\mathrm{kg} / \mathrm{fed})$ ) and five foliar treatments of ( (tap water), Ca (calcium citrate $2.5 \mathrm{mg} / \mathrm{L})+\mathrm{B}$ (boric acid $1.5 \mathrm{mg} / \mathrm{L})$, Yeast $(10 \mathrm{mg} / \mathrm{L})+\mathrm{K}$ (potassium citrate $2.5 \mathrm{mg} / \mathrm{L})$, Yeast $(10$ $\mathrm{mg} / \mathrm{L}$ ) + NAA (naphthalene acetic acid $2.5 \mathrm{mg} / \mathrm{L})+\mathrm{K}$ (potassium citrate $4 \mathrm{mg} / \mathrm{L}$ ) and $\mathrm{Zn}(0.35 \mathrm{gm} / 100 \mathrm{~L})+\mathrm{Cu}(0.20 \mathrm{gm} / 100 \mathrm{~L})+\mathrm{Mn}(0.25 \mathrm{gm} / 100 \mathrm{~L})$ in sulphure form $)$. Each treatment was replicated three times. Thus, the total numbers of plots were 45 plots plus control treatment. The NPK fertilizers were added to soil cultivated with pepper plants as recommended by the Ministry of Agriculture and Soil Reclamation. The seedlings of bell pepper (Capsicum annuum L.) were transplanted on $1^{\text {st }}$ of April in 2012 and 2013, respectively, on two side of ridges at $30 \mathrm{~cm}$ a part.

Plant height, fresh weight, dry weight, leaf area, number of leaves, number of flowers and number of branches were measured as vegetative growth parameters. Yield and its components as number of fruits, fruit weight $\mathrm{g} / \mathrm{plant}$, earl and total yield (ton/fed.). as for fruit quality Chlorophylls (a, b and total chlorophyll), $\mathrm{NO}_{2}-\mathrm{N}(\mathrm{mg} / \mathrm{kg})$, Vitamin C $(\mathrm{mg} / 100 \mathrm{~g})$, Acidity \%, Total carbohydrate (\%), Xanthophyll and carotene $(\mathrm{mg} / 100 \mathrm{~g})$, TSS \% reduce sugar, non-reduce sugar and total sugar \%.. The highest values of all parameters were recorded with spray $\mathrm{Y}+\mathrm{NAA}+\mathrm{K}$ with adding humic acid at $5 \mathrm{~kg} / \mathrm{fed}$. From that result it can concluded that the treatment of adding $5 \mathrm{~kg} / \mathrm{fed}$. humic acid with spray $\mathrm{Y}+\mathrm{NAA}+\mathrm{K}$ followed by the spray treatment of $\mathrm{Zn}+\mathrm{Cu}+\mathrm{Mn}$ under the same soil application considered the best combination and it is recommended for pepper plant grown under similar field conditions.

Keywords :soil application, humic acid, foliar application,(yeast), vegetative growth,yield of pepper, pre harvesting and pepper plant.
\end{abstract}

\section{INTRODUCTION}

Bell pepper (Capsicum annuum L.) is an important vegetable crop used throughout the world. Increasing plant high yeild, depend on many factors, Organic materials such as straw rice costs are available in abundance and reach tremendous amounts every day.Straw rice costs is commonly applied to the soils to improve their physical, chemical and biological properties.

Humic acid is one of the major components of humic substances. Humic substances have a very profound influence on plant growth, either 
directly or indirectly. The indirect effects of humic compounds on soil fertility include; increasing soil microbial population including beneficial microorganisms, improving soil structure and increasing in the cation exchange capacity (CEC) and $\mathrm{pH}$ buffering capacity of the soil. increased photosynthesis and respiration rates in plants, enhanced protein synthesis and enhancing the uptake of minerals through the stimulation of microbiological activity. (Anonymous, 2010).

\begin{tabular}{|c|c|c|c|}
\hline 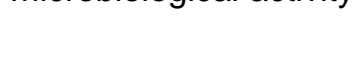 & HUMIN & HUMIC ACID & FULVIC ACID \\
\hline $\begin{array}{l}\text { Molecularweight } \\
\text { decreasing }\end{array}$ & 100,000 & 10,000 & 1,000 \\
\hline $\begin{array}{l}\text { Cation exchange } \\
\text { capacity and } \\
\text { acidity }(\mathrm{mol} / \mathrm{kg})\end{array}$ & 300 & 500 & 1,000 \\
\hline Carbon content $(\mathrm{g} / \mathrm{kg})$ & 620 & 520 & 430 \\
\hline $\begin{array}{l}\text { Oxygen } \\
\text { content }(\mathrm{g} / \mathrm{kg})\end{array}$ & 290 & 440 & 510 \\
\hline $\begin{array}{l}\text { Nitrogen } \\
\text { content }(\mathrm{g} / \mathrm{kg})\end{array}$ & 55 & 43 & 7 \\
\hline $\begin{array}{l}\text { Hydrogen } \\
\text { content }(\mathrm{g} / \mathrm{kg})\end{array}$ & 29 & 33 & \\
\hline
\end{tabular}

Hydrophilic polyemers exist in three types including; natural (polysaccharide derivatives), semi artificial (cellulosic primitive derivatives) and artificial (Mikkelsen, 1999). Artificial polymers used more than natural ones, because has more stability against environmental break down (Peterson, 2002). Super absorbent polymers do not treat human life and environment (Boatright et al., 1997). Classification of polymers:

Basis of Classification
Origin
Thermal Response
Mode of formation
Line structure
Properties
Tacticity
Crystallinity
Polarity

\author{
polymer type \\ natural,Semi synthetic,Synthetic \\ Thermoplastic, Thermosetting \\ Addition, Conedensation \\ Linear,Branched \\ Rubber,Plastic,Fiber \\ Isotactic, Syndiotactis, Atactic \\ Semi-crystalline, Crystalline \\ Polar, Non polar
}

Foliar spray with micronutrients usually penetrates through the cuticle of leaves or the stomata then enters the cells and results in higher fruit and seed yield. Borax contains about $16 \%$ Boron, a micronutrient which is easily leached from the soil. Boron has several roles on growth and development of plants such as branches, and fruit development as well as stamen fertility. Zinc is a micronutrient necessary for plant growth. It promotes growth hormone biosynthesis, the formation of starch and seed production and maturation, Manganese an essential micronutrient for all higher plant it 
participates in the structure of photosynthetic proteins and enzymes. Copper is essential to complete the plants life cycle. Copper occurs in enzymatic composition of vital importance in plant metabolism and participates in the photosynthesis, respiration, carbohydrate metabolism.

Foliar application of yeast extract plays an important role in soil biofertility because of its capability for producing hormones, amino acids, cytokinin, indole and vitamins. Also, foliar application of NAA significantly increased fruit yield, number of fruits and average fruit weight (AFW) of bell pepper (Sridhar et al., 2009).

Therefore, the present investigation was designed to determine the suitable treatments of straw rice costs, humic acid, polymers, some macro and micro-elements, yeast, and naphthalene acetic acid to obtain high productivity and fruit quality of pepper plants.

\section{MATERIALS AND METHODS}

Two field experiments were carried out at El-Baramon experimental farm, Dakahlia Governorate, Egypt, during the two summer seasons of 2012 and 2013 to investigate the effect of treatments as well as their interactions on growth and productivity of bell pepper (Capsicum annuumL.) variety of " California wonder".

Fifteen treatments were arranged in split plots design, which were the simple possible combination between:

\section{A- Soil application which all added before transplanting}

1. (Straw rice costs (4 ton/fed.),

2. Humic acid $(5 \mathrm{~kg} / \mathrm{fed}$.),

3. Polymers $(20 \mathrm{~kg} / \mathrm{fed}))$.

B - Five foliar treatments as sub plot :

1. Tap water .

2. calcium citrate $(2.5 \mathrm{mg} / \mathrm{L})+$ boric acid $(1.5 \mathrm{mg} / \mathrm{L})$,

3. Yeast $(10 \mathrm{mg} / \mathrm{L})+$ potassium citrate $(2.5 \mathrm{mg} / \mathrm{l})$,

4. Yeast $(10 \mathrm{mg} / \mathrm{L})+\mathrm{NAA}$ naphthalene acetic acid $(2.5 \mathrm{mg} / \mathrm{L})+$ potassium citrate $(4 \mathrm{mg} / \mathrm{L})$

5. Zn $(0.35 \mathrm{gm} / 100 \mathrm{~L})+\mathrm{Cu}(0.20 \mathrm{gm} / 100 \mathrm{~L})+\mathrm{Mn}(0.25 \mathrm{gm} / 100 \mathrm{~L})$ in sulphure form.

Each treatment was replicated three times. Thus, the total numbers of plots were 45 plots plus control treatment. The area of experiment plot was $30 \mathrm{~m}^{2}$ and each plot included (10 ridges ridge was $1.00 \mathrm{~m}$ in width and $3 \mathrm{~m}$ length).the foliar application sprayed three times at 45 days after planting and repeated every 15 days interval.

Representative samples were collected from the surface layer $(0-30$ $\mathrm{cm}$ ) of the experimental soil and analyzed for some physical and chemical properties as shown in Table (1). 
Table 1: Some physical and chemical properties of the experimental soil during both seasons of 2012 and 2013.

\begin{tabular}{|l|c|c|c|c|c|c|c|c|c|c|c|}
\hline \multirow{2}{*}{ Season } & \multicolumn{4}{|c|}{ Physical properties\% } & \multicolumn{4}{c|}{ Chemical properties } \\
\cline { 2 - 11 } & clay & silt & $\begin{array}{c}\text { Fine } \\
\text { sand }\end{array}$ & $\begin{array}{c}\text { Coarse } \\
\text { sand }\end{array}$ & $\begin{array}{c}\text { Texture } \\
\text { class }\end{array}$ & $\mathbf{O} . \mathbf{M} \%$ & $\begin{array}{c}\mathbf{N} \\
\mathbf{p p m}\end{array}$ & $\begin{array}{c}\mathbf{P} \\
\mathbf{p p m}\end{array}$ & $\begin{array}{c}\mathbf{K} \\
\mathbf{p p m}\end{array}$ & $\mathbf{p H}$ & $\begin{array}{c}\text { ECds/ } \\
\mathbf{m}\end{array}$ \\
\hline 2012 & 66.82 & 17.68 & 12.95 & 2.55 & clay & 2.52 & 32.50 & 6.02 & 340 & 7.79 & 1.47 \\
\hline 2013 & 66.74 & 17.71 & 12.97 & 2.58 & clay & 2.49 & 33.01 & 6.08 & 348 & 7.84 & 1.52 \\
\hline
\end{tabular}

- EC: electrical conductivity $\mathrm{pH}$ : soil reaction $\quad$ O.M: organic matter

Yeast extract was prepared from brewer's, dissolved in water followed by adding sugar at a ratio of $1: 1$ and kept 24 hours in a warm place for reproduction according to the methods of Morsi et al., (2008). Chemical analysis of activated yeast is shown in Table (2).

The seedlings of bell pepper (Capsicum annuumL.) were transplanted on $1^{\text {st }}$ of April in 2012 and 2013, on one side of ridges at $30 \mathrm{~cm}$ a part.

During the growing seasons, the NPK fertilizers were added to soil cultivated with pepper plants as recommended by the Ministry of Agriculture and Soil Reclamation, $200 \mathrm{~kg} / \mathrm{fed} \mathrm{N}$ as ammonium sulphate $(20.5 \% \mathrm{~N}), 150$ $\mathrm{kg} / \mathrm{fed} \mathrm{P}_{2} \mathrm{O}_{5}$ as super phosphate $\left(15.5 \% \mathrm{P}_{2} \mathrm{O}_{5}\right)$ and $50 \mathrm{~K} \mathrm{~kg} / \mathrm{fed}$ as Potassium sulphate $\left(48 \% \mathrm{~K}_{2} \mathrm{O}\right)$. Nitrogen was added after one month from transplanting, Phosphorus fertilizer was added to the soil after two months from planting, while $\mathrm{K}$ fertilizers were added in two dose; before and after flowering date.

Table 2: Chemical analysis of yeast used.

\begin{tabular}{|l|c|l|r|l|c|}
\hline \multicolumn{2}{|c|}{ Minerals mg } & \multicolumn{2}{c|}{ Amino acids mg } & \multicolumn{2}{c|}{ Vitamins mg } \\
\hline Total N & 7.23 & Arginine & 1.99 & Thiamin & 2.71 \\
\hline $\mathrm{P}_{2} \mathrm{O}_{5}$ & 51.68 & Hustidine & 2.63 & Riboflavin & 4.96 \\
\hline $\mathrm{K} 2 \mathrm{O}$ & 34.39 & lsoleiucine & 2.31 & Nicotinic acid & 39.88 \\
\hline $\mathrm{MgO}$ & 5.76 & Leucine & 3.09 & Pantothenic acid & 19.56 \\
\hline $\mathrm{CaO}$ & 3.05 & Lysine & 2.95 & Biotin & 0.09 \\
\hline $\mathrm{SiO}_{2}$ & 1.55 & Methionine & 0.72 & Pyridoxine & 2.90 \\
\hline $\mathrm{SO}_{2}$ & 0.49 & Phyrnylalanine & 2.01 & Folic acid & 4.36 \\
\hline $\mathrm{NaCl}$ & 0.30 & Threonine & 2.09 & Cobalamin & 153 \\
\hline $\mathrm{Fe}$ & 0.92 & Tryptophan & 0.45 & Enzymes & ug \\
\hline $\mathrm{Ba}$ & 157.6 & Valine & 2.19 & Oxidase & 0.350 \\
\hline $\mathrm{Co}$ & 67.8 & Glutamic acid & 2.00 & Catalase & 0.290 \\
\hline $\mathrm{Pd}$ & 438.6 & Serine & 1.59 & & \\
\hline $\mathrm{Mn}$ & 81.3 & Aspartic acid & 1.33 & & \\
\hline $\mathrm{Sn}$ & 223.9 & Praline & 1.53 & & 23.20 \\
\hline $\mathrm{Zn}$ & 335.6 & Tyrosine & 1.49 & Carbohydraytes & \\
\hline $\mathrm{Data}$ & & & & &
\end{tabular}

\section{Data recorded:}

\section{A. Vegetative growth characteristics :}

Three plants were randomly taken from each treatment after 85 days from transplanting) and the vegetative growth parameters were measured in expression of, Plant height $(\mathrm{cm})$, No. of leaves/plant, No. of flowers/plant, No. of branches/plant, Fresh and dry weight of plant (gm/plant) as well as Leaf area /plant $\left(\mathrm{cm}^{2}\right)$ Leaf area was calculated according to the formula described by Koller (1972) as follows: 


\section{Area of the disks $\left(\mathrm{cm}^{2}\right) \square$ Dry weight of leaves/plant}

Total leaf area $=$

\section{B. Chemical quality:}

\section{Dry weight of disks}

Representative samples of pepper fruits were randomly taken from each treatment at the fourth picking to determine the quality parameters of pepper fruits and were expressed as follows: Chlorophylls ( $a, b$ and total chlorophyll,.Goodwine(1965), $\quad \mathrm{NO}_{2}-\mathrm{N}$ (mg/kg), Singh(1988), Vitamin C (mg/100g), Acidity \%, Total carbohydrate (\%).,(A.O.C.,2000), Xanthophyll and carotene $(\mathrm{mg} / 100 \mathrm{~g})$ Dubois et al.(1956, and Jackson(1967),, TSS \% .,(A.O.C.,2000), reduce sugar, non-reduce sugar and total sugar\% Sadasivamand Manickam,(1996). Random samples of pepper fruits were randomly chosen from each treatment, oven dried at $70^{\circ} \mathrm{C}$ and ground for the determination of $\mathrm{N}, \mathrm{P}$ and $\mathrm{K}$ contents respectively.

\section{Fruit Yield and its components:}

Ten picking with 7 days intervals were harvested starting after 90 days from transplanting. The following data were recorded $d$ : fruit weight $(\mathrm{g})$, no of fruits/plant, early yield (ton/fed) and total yield (ton/fed) :data of the first three picking in two seasons were calculated.

\section{Methods of analysis:}

Determination of quality parameters in pepper fruits:

- Chlorophyll content was estimated as the method described by Goodwine (1965)

- Total carotenoides were described by Dubois et al. (1956)

- Nitrate content in pepper fruits were according to the method described by Singh (1988).

- Total soluble solids (TSS \%) was estimated using Galli 110 refractometer according to (A.O.A.C., 2000)

- Ascorbic acid (vitamin $C$ ) in pepper fruits was determined by titration with 2.6 diclorophenol indophenol blue dye according to the method reported in (A.O.A.C., 2000).

- Total soluble sugar, was determined according to the method described by Sadasivam and Manickam, (1996).

- Reducing sugar was estimated by Nelson-Somogy method as described by Naguib (1964).

- The acidity in the fruits juice was according to (A.O.A.C., 2000).

- Xanthophyll in pepper fruit was determined using spectrophotometer at wave length 474 n.m as described by (A.O.A.C., 2000).

- For estimation of total carbohydrates in fresh fruits; The green colours was measured spectrophotometrically at $630 \mathrm{~nm}$. Sadasivam and Manickam, (1996).

All data were statistically analyzed according to the technique of analysis variance (ANOVA) and the least significant difference (L.S.D) method was used to compare the deference between the means of treatment values according to the methods described by Gomez and Gomez, (1984). All 
statistical analysis were performed using analysis of variance technique by means of CoSTATE Computer Software.

\section{RESULTS AND DISCUSSION}

\section{Vegetative growth and flowering parameters}

Data of vegetative growth parameters, i.e., plant height, fresh weight, dry weight, leaf area/plant, number of leaves, number of flowers, and number of branches/ plant affected by individual treatments as well as their interaction are president in Tables 3 and 4.

\section{Effect of soil application:}

Concerning the effect of soil treatments; data in Table 3 and 4 revealed that, the mean values of plant height ,fresh weight, dry weight, leaf area/plant ,number of leaves, flowers and branches/ plant of pepper plant were significantly increased due to adding humic acid and polymers during both seasons of the study.

Data presented in Tables 3 and 4, show that adding humic acid gave the highest values of the vegetative parameters during both seasons followed by polymers except fresh weight in the second season and dry weight in both season and finally straw rice costs. Generally, adding $5 \mathrm{~kg} / \mathrm{fed}$. humic acid was superior one for enhancing plant height ,fresh weight ,dry weight , leaf area/plant, number of leaves, number of flower and number of branches/ plant. Increases in the vegetative growth of pepper plant by adding humic acid might be referred to positive mineral effect and also hormone like NAA activate on vegetative growth according to Abdel Fatah et al., (2008), Eyheraguibel et al., (2008) and Mahmoud and Hafez, (2010) showed that the vegetative growth parameters of potato yield and tuber quality as well as nutritive value of potato tuber were significantly increased with increasing the level of humic acid application.

\section{Effect of foliar application:}

Regarding to the effect of foliar application of some substants on vegetative growth parameters data in Tables 3 and 4 indicated that, the mean values of parameters under study were significantly increased with increasing foliar application during both seasons. On the other hand, the highest values of plant height, fresh weight, dry weight, leaf area/plant, number of leaves, flowers and branches/ plant of pepper plant were realized when the plants sprayed with $\mathrm{Y}+\mathrm{NAA}+\mathrm{K}$ and followed by $\mathrm{Y}+\mathrm{K}$ compared with the untreated plants. The same trend was true during the second season except fresh weight in the both seasons.

Kazemi (2013) studied the effects of foliar application of humic acid and potassium nitrate on vegetative and reproductive growth, yield and quality of cucumber plants. Humic acid (20 and $40 \mathrm{ppm}$ ) and potassium nitrate (100 and $200 \mathrm{mg} / \mathrm{L}$ ) solutions were applied as foliar sprays. Results indicated that humic acid and potassium nitrate increased vegetative and reproductive growth by increasing both plant height and dry weights and yield. These results agreed with those obtained by El-Tohamy et al., (2008) on egg-plant and Sridhar et al., (2009) as for the effect of foliar application on bell pepper, 
mentioned that yeast extract and naphthalene acetic acid (NAA) increased plant height, number of branches, leaves per plant and dry weight as well. These results may be due to the fact that yeast extract contains growth factors and a relatively larger proportion of free amino acids and short peptides of two or three amino acids longer than protein hydrolisates Bevilacqua et al., 2008. Also, Fawzy et al., (2005), El-Bassiony et al., (2012) and Bhuvaneswari et al. (2013) on sweet pepper resulted that increasing plant vegetative growth due to increasing potassium fertilization levels.

Table (3): Effect of soil and foliar application as well as their interaction on plant height fresh weight, dry weight, and leaf area/plant of pepper plant during 2012 and 2013 seasons.

\begin{tabular}{|c|c|c|c|c|c|c|c|c|c|}
\hline \multirow{2}{*}{\multicolumn{2}{|c|}{ Char }} & \multicolumn{2}{|c|}{$\begin{array}{l}\text { Plant height } \\
\text { (cm) }\end{array}$} & \multicolumn{2}{|c|}{$\begin{array}{l}\text { Fresh weight } \\
\text { (g) }\end{array}$} & \multicolumn{2}{|c|}{$\begin{array}{l}\text { Dry weight } \\
\text { (g) }\end{array}$} & \multicolumn{2}{|c|}{$\begin{array}{c}\text { Leaf area/plant } \\
\left(\mathrm{cm}^{2}\right)\end{array}$} \\
\hline & & & & $1^{\text {st }}$ & $2^{\text {nd }}$ & & & & \\
\hline \multicolumn{10}{|c|}{ A:Soil application } \\
\hline \multicolumn{2}{|l|}{ S.R } & 30.42 & 28.31 & 729.19 & 781.29 & 125.49 & 135.41 & 84.05 & 90.92 \\
\hline \multirow{2}{*}{\multicolumn{2}{|c|}{$\begin{array}{l}\text { H.A } \\
\text { Poly. }\end{array}$}} & 42.50 & 39.37 & 756.76 & 826.20 & 139.42 & 145.16 & 117.42 & 126.45 \\
\hline \multirow{2}{*}{\multicolumn{2}{|c|}{$\begin{array}{l}\text { Poly. } \\
\text { LSD at } 5 \%\end{array}$}} & 36.31 & 33.71 & 733.55 & 752.12 & 110.33 & 125.40 & 100.30 & 108.25 \\
\hline & & 0.42 & 0.64 & 4.59 & 2.00 & 2.49 & 1.64 & 1.20 & 2.05 \\
\hline \multicolumn{2}{|c|}{ LSD at $5 \%$} & & \multicolumn{7}{|c|}{ B:Foliar application } \\
\hline \multicolumn{2}{|c|}{ control } & 35.02 & 32.69 & 716.77 & 759.90 & 121.32 & 123.98 & 96.74 & 104.98 \\
\hline \multicolumn{2}{|c|}{$\mathrm{Ca}+\mathrm{B}$} & 37.75 & 34.97 & 730.53 & 778.59 & 125.32 & 135.04 & 104.29 & 112.31 \\
\hline \multirow{2}{*}{\multicolumn{2}{|c|}{$\frac{Y+K}{Y+N A A+K}$}} & 38.14 & 35.29 & 736.82 & 787.20 & 128.12 & 140.72 & 105.36 & 113.35 \\
\hline & & 40.73 & 37.80 & 750.18 & 798.46 & 131.98 & 143.29 & 112.52 & 121.39 \\
\hline \multicolumn{2}{|c|}{$Z n+C u+M n$} & 30.42 & 28.24 & 764.87 & 808.54 & 118.65 & 133.58 & 84.03 & 90.68 \\
\hline \multicolumn{2}{|c|}{ LSD at $5 \%$} & 0.35 & 0.43 & 7.35 & 3.86 & 2.84 & 1.72 & 0.94 & 1.38 \\
\hline \multicolumn{10}{|c|}{ A×B:Interaction } \\
\hline \multirow{5}{*}{ S.R } & cont & 28.26 & 26.32 & 716.57 & 753.27 & 21.87 & 126.50 & 78.06 & 84.53 \\
\hline & $\mathrm{Ca}+\mathrm{B}$ & 29.50 & 27.76 & 723.33 & 774.83 & 125.10 & 137.10 & 81.49 & 89.14 \\
\hline & $\mathrm{Y}+\mathrm{K}$ & 31.33 & 29.06 & 737.47 & 783.43 & 128.73 & 141.23 & 86.54 & 93.32 \\
\hline & $\mathrm{Y}+\mathrm{NAA}$ & 33.27 & 30.79 & 748.17 & 801.77 & 131.20 & 141.90 & 91.91 & 98.90 \\
\hline & $\mathrm{Zn}+\mathrm{Cu}+\mathrm{Mn}$ & 29.77 & 27.63 & 720.41 & 793.17 & 120.54 & 130.30 & 82.24 & 88.71 \\
\hline \multirow{5}{*}{ H.A } & & 41.67 & 39.15 & 755.90 & 812.27 & 138.37 & 135.97 & 115.10 & 125.72 \\
\hline & $\mathrm{Ca}+\mathrm{B}$ & 46.95 & 42.96 & 782.80 & 829.87 & 138.33 & 141.20 & 129.70 & 137.94 \\
\hline & $Y+K$ & 44.11 & 40.68 & 773.53 & 829.27 & 143.50 & 152.33 & 121.86 & 130.65 \\
\hline & $\mathrm{Y}+\mathrm{NAA+}$ & 48.89 & 45.39 & 793.17 & 835.17 & 149.20 & 159.57 & 135.07 & 145.78 \\
\hline & $\mathrm{Zn}+\mathrm{Cu}+\mathrm{Mn}$ & 30.90 & 28.69 & 678.40 & 824.43 & 127.71 & 136.73 & 85.36 & 92.14 \\
\hline \multirow{5}{*}{ Poly. } & control & 35.14 & 32.60 & 677.83 & 714.17 & 103.73 & 109.47 & 97.06 & 104.69 \\
\hline & $\mathrm{Ca}+\mathrm{B}$ & 36.81 & 34.20 & 685.47 & 731.07 & 112.53 & 126.83 & 101.68 & 109.84 \\
\hline & $Y+K$ & 38.98 & 36.14 & 699.47 & 748.90 & 112.13 & 128.60 & 107.69 & 116.06 \\
\hline & $Y+\mathrm{NA}$ & 40.03 & 37.20 & 709.20 & 758.43 & 115.53 & 128.40 & 110.59 & 119.50 \\
\hline & $\mathrm{Zn}+\mathrm{Cu}+\mathrm{Mn}$ & 30.59 & 28.39 & 895.80 & 808.03 & 107.70 & 133.70 & 84.50 & 91.18 \\
\hline \multicolumn{2}{|c|}{ LSD at $5 \%$} & 0.60 & 0.74 & 12.74 & 6.69 & \begin{tabular}{|l|}
4.92 \\
\end{tabular} & 2.98 & 7.99 & 2.38 \\
\hline
\end{tabular}

S.R: Straw rice costs H.A: humic acid poly: polymers Ca: calcium citrate

B: Borax Y: yeast NAA: naphthalene acetic acid

$\mathrm{K}$ : potassium citrate $\mathrm{Zn}+\mathrm{Cu}+\mathrm{Mn}$ : micronutrient 
Effect of interaction between soil and foliar application:

The effect of interaction between soil and foliar application on plant height, fresh weight, dry weight, leaf area/plant, number of leaves, flowers and branches / plantmof pepper plant are present in Tables 3 and 4. It could be indicated that; the average values of all studied growth parameters were significantly affected by the addition of all investigated treatments. Such effect was more pronounced for the treatment of humic acid with all foliar application comparing with the control, which gave the lowest values. In this connect, the highest mean values; plant height, fresh weight, dry weight, leaf area/plant, number of leaves, flowers and branches/ plant, respectively were recorded when the plants treated with adding humic acid with spray of $\mathrm{Y}+\mathrm{NAA}+\mathrm{K}$

Table (4): Effect of soil and foliar application as well as their interaction on number of leaves, flowers, and branches/ plant of pepper plant during 2012 and 2013 seasons.

\begin{tabular}{|c|c|c|c|c|c|c|c|}
\hline \multirow{2}{*}{ Treat. } & \multirow[t]{2}{*}{ Char. } & \multicolumn{2}{|c|}{$\begin{array}{c}\text { No. of } \\
\text { leaves/plant }\end{array}$} & \multicolumn{2}{|c|}{$\begin{array}{l}\text { No. of flowers } \\
\text { /plant }\end{array}$} & \multicolumn{2}{|c|}{$\begin{array}{c}\text { No. of } \\
\text { branches/plant }\end{array}$} \\
\hline & & & $2^{\text {nd }}$ & & $2^{\text {nd }}$ & & \\
\hline \multicolumn{8}{|c|}{ A:Soil application } \\
\hline S.R & & 29.82 & 33.80 & 15.24 & 15.57 & 5.48 & 5.51 \\
\hline H.A & & 41.67 & 46.60 & 17.33 & 17.74 & 7.50 & 7.66 \\
\hline Poly. & & 35.59 & 39.53 & 16.26 & 16.63 & 6.47 & 6.56 \\
\hline $\mathrm{LSD}_{\text {at }}$ & & 0.51 & 1.71 & 0.08 & 0.12 & 0.29 & 0.24 \\
\hline \multicolumn{8}{|c|}{ B:Foliar application } \\
\hline \multicolumn{2}{|c|}{ Control } & 34.33 & 39.00 & 16.04 & 16.43 & 6.31 & 6.36 \\
\hline \multirow{2}{*}{\multicolumn{2}{|c|}{\begin{tabular}{|l|}
$\mathrm{Ca}+\mathrm{B}$ \\
$\mathrm{Y}+\mathrm{K}$
\end{tabular}}} & 37.01 & 41.33 & 16.51 & 16.88 & 6.80 & 6.81 \\
\hline & & 37.39 & 41.78 & 16.57 & 16.94 & 6.76 & 6.87 \\
\hline \multicolumn{2}{|c|}{$\mathrm{Y}+\mathrm{NAA}+\mathrm{K}$} & 39.93 & 45.00 & 17.02 & 17.43 & 7.34 & 7.36 \\
\hline \multirow{2}{*}{\multicolumn{2}{|c|}{$\mathrm{Zn}+\mathrm{Cu}+\mathrm{Mn}$}} & 29.82 & 32.78 & 15.24 & 15.55 & 5.23 & 5.49 \\
\hline \multirow{2}{*}{\multicolumn{2}{|c|}{ LSD at $5 \%$}} & 0.34 & 1.35 & 0.06 & 0.08 & 0.25 & 0.21 \\
\hline & & \multicolumn{6}{|c|}{ AXB:Interaction } \\
\hline \multirow{5}{*}{ S.R } & Control & 27.70 & 31.33 & 14.87 & 15.17 & 5.09 & 5.12 \\
\hline & $\mathrm{Ca}+\mathrm{B}$ & 28.92 & 33.00 & 15.09 & 15.46 & 5.31 & 5.40 \\
\hline & $Y+K$ & 30.71 & 34.67 & 15.40 & 15.71 & 5.64 & 5.66 \\
\hline & $\mathrm{Y}+\mathrm{NAA}+\mathrm{K}$ & 32.61 & 37.00 & 15.74 & 16.05 & 5.99 & 5.99 \\
\hline & $\mathrm{Zn}+\mathrm{Cu}+\mathrm{Mn}$ & 29.18 & 33.00 & 15.13 & 15.43 & 5.36 & 5.38 \\
\hline \multirow{5}{*}{ H.A } & Control & 40.84 & 46.33 & 17.18 & 17.70 & 7.50 & 7.62 \\
\hline & $\mathrm{Ca}+\mathrm{B}$ & 46.02 & 51.00 & 18.09 & 18.44 & 8.46 & 8.36 \\
\hline & $Y+K$ & 43.24 & 49.00 & 17.60 & 18.00 & 7.95 & 7.92 \\
\hline & $\mathrm{Y}+\mathrm{NAA}+\mathrm{K}$ & 47.93 & 54.33 & 18.43 & 18.92 & 8.81 & 8.83 \\
\hline & $\mathrm{Zn}+\mathrm{Cu}+\mathrm{Mn}$ & 30.29 & 32.33 & 15.33 & 15.64 & 4.81 & 5.58 \\
\hline \multirow{5}{*}{ Poly. } & Control & 34.44 & 39.33 & 16.06 & 16.41 & 6.33 & 6.34 \\
\hline & $\mathrm{Ca}+\mathrm{B}$ & 36.08 & 40.00 & 16.34 & 16.72 & 6.63 & 6.66 \\
\hline & $\mathrm{Y}+\mathrm{K}$ & 38.21 & 41.67 & 16.72 & 17.11 & 6.70 & 7.03 \\
\hline & $\mathrm{Y}+\mathrm{NAA}+\mathrm{K}$ & 39.24 & 43.67 & 16.90 & 17.32 & 7.21 & 7.24 \\
\hline & $\mathrm{Zn}+\mathrm{Cu}+\mathrm{Mn}$ & 29.98 & 33.00 & 15.27 & 15.58 & 5.51 & 5.53 \\
\hline \multicolumn{2}{|c|}{$\mathrm{LSD}_{\text {at5\% }}$} & 0.59 & 2.33 & 0.10 & 0.15 & 0.42 & 0.36 \\
\hline
\end{tabular}




\section{Yield and its components}

Yield and its components could be considered to be the mirror of all growth features. The results given in Table 5 present the response of yield and its components of pepper plant, i.e., total yield (ton/fed), early yield (ton/fed), number of fruits/plant, and fruit weight (g/plant) to soil application, foliar application and its interaction.

\section{Effect of soil application:}

In this study a comparing of the effect of soil application of some substances on yield and its components of pepper plant, the presented results in Table 5 show that the application of soil materials significantly increased total yield (ton/fed), early yield (ton/fed), number of fruits/plant and fruits weight ( $\mathrm{g} / \mathrm{plant})$ during both seasons. Pepper plant fertilized with humic acid at the rate $5 \mathrm{~kg} / \mathrm{fed}$. recorded the highest values of parameters under study, while the lowest ones recorded with adding fine rice straw.

The obtained results are in agreement with those recorded by Karakurt et al., (2009) who demonstrated that humic acid applications might successfully be used to obtain higher fruit yield in organically grown pepper. Moreover, Arancon et al., (2006) reported that pepper plants treated with humic acid significantly produced more fruits and flowers than untreated plants. In addition, Padem and Ocal (1999) demonstrated that increasing K-humate application dose led to a significant increase in fruit weight and total yield.

\section{Effect of foliar application:}

Concerning to the effect of the foliar spray of some substances, it was found as shown in Table 5 that foliar application significantly enhanced the parameters of total yield (ton/fed), early yield (ton/fed), number of fruits/plant, and fruits weight ( $\mathrm{g} / \mathrm{plant}$ ) during both seasons. The highest values of the parameters under investigation recorded with using $\mathrm{Y}+\mathrm{NAA}+\mathrm{K}$ during the both seasons.

As for the effect of foliar application of some substances, the positive effects of applying active dry yeast was attributed to its own contents of different nutrients, high percentage of protein, large amounts of vitamin $B$ and natural plant growth regulators such as cytokinins (Fathy and Farid, 1996). Physiological roles of vitamins and amino acids in the yeast extract which increased the metabolic processes role and levels of endogenous hormones, i.e., IAA and GA3 (Sarhan and Abdullah, 2010) and may promoted the vegetative growth characters which in turn reflected on increasing the fruit yield. Similar trend of results were reported by Gomaa et al., (2005) on potao; El-Tohamy et al., (2008) on eggplant; Shehata et al., (2012)on cucumber plant. Also, Alam and Khan, (2002) on tomato plant; Sridhar et al., (2009) on bell pepper, and Rongsennungla et al., (2011) resulted that highest fresh yield and cured fruit yield were also obtained from NAA at $40 \mathrm{ppm}$ which was significantly higher than other treatments. On the other hand, for effect of potassium on plant similar result reported by Majumdar et al., (2000) on tomato; Fawzy et al., (2005) on sweet pepper, and El-Bassiony et al., (2012) investigated the response of sweet pepper plants to different rates of potassium fertilization. The highest potassium fertilization rate $(200 \mathrm{~kg} / \mathrm{fed}$.) gave the highest total yield. 
Effect of interaction between soil and foliar application:

Response of total yield (ton/fed), early yield (ton/fed), number of fruits/plant, and fruit weight ( $\mathrm{g} / \mathrm{plant}$ ) of pepper plant to the interaction effect of soil and foliar application, were found to be increased significantly with using foliar spray under all soil application in two successive seasons. The highest values of all parameters were recorded with spray $\mathrm{Y}+\mathrm{NAA}+\mathrm{K}$ with adding humic acid at $5 \mathrm{~kg} / \mathrm{fed}$. followed by using any foliar application under the same soil application compared with the untreated plant during both seasons.

Table (5): Effect of soil and foliar application of some substances as well as their interaction on number of fruit, fruit weight, early yield and total yield of pepper plant during 2012 and 2013 seasons.

\begin{tabular}{|c|c|c|c|c|c|c|c|c|c|}
\hline \multirow[t]{2}{*}{ Treat. } & \multirow[t]{2}{*}{ Char. } & \multicolumn{2}{|c|}{$\begin{array}{c}\text { No. of } \\
\text { fruit/plant }\end{array}$} & \multicolumn{2}{|c|}{$\begin{array}{c}\text { fruit } \\
\text { weight/(pla } \\
\text { nt) }\end{array}$} & \multicolumn{2}{|c|}{$\begin{array}{c}\text { Early yield } \\
\text { (ton/fed.) }\end{array}$} & \multicolumn{2}{|c|}{$\begin{array}{l}\text { Total yield } \\
\text { (ton/fed.) }\end{array}$} \\
\hline & & $1^{\text {st }}$ & $2^{\text {nd }}$ & $1^{\text {st }}$ & $2^{\text {nd }}$ & $1^{\mathrm{st}}$ & $2^{\text {nd }}$ & $1^{\mathrm{st}}$ & $2^{\text {nd }}$ \\
\hline \multicolumn{10}{|c|}{ A:Soil application } \\
\hline S.R & & 10.05 & 9.43 & 481.3 & 484.2 & 3.452 & 4.506 & 9.421 & 9.511 \\
\hline H.A & & 13.46 & 12.66 & 672.4 & 673.2 & 7.0068 & 7.0207 & 15.344 & 15.367 \\
\hline Poly. & & 11.46 & 10.90 & 574.4 & 575.7 & 5.184 & 5.208 & 12.306 & 12.348 \\
\hline $\operatorname{LSD}_{\mathrm{f}}$ & or $5 \%$ & 0.76 & 1.49 & 6.88 & 7.08 & 127.89 & 131.69 & 213.16 & 219.47 \\
\hline \multicolumn{10}{|c|}{ B:Foliar application } \\
\hline Contr & & 11.24 & 10.51 & 554.0 & 557.4 & 4.804 & 4.867 & 11.673 & 11.778 \\
\hline $\mathrm{Ca}+\mathrm{B}$ & & 12.41 & 11.60 & 597.2 & 598.2 & 5.608 & 5.625 & 13.0146 & 13.0429 \\
\hline $\mathrm{Y}+\mathrm{K}$ & & 12.17 & 11.45 & 603.4 & 604.8 & 5.722 & 5.748 & 13.204 & 13.247 \\
\hline $\mathrm{Y}+\mathrm{NA}$ & $\mathrm{A}+\mathrm{K}$ & 12.87 & 12.30 & 644.4 & 646.0 & 6.485 & 6.516 & 14.475 & 14.527 \\
\hline $\mathrm{Zn}+\mathrm{C}$ & $\mathrm{u}+\mathrm{Mn}$ & 9.58 & 9.13 & 481.2 & 482.2 & 3.450 & 3.469 & 9.418 & 9.449 \\
\hline $\operatorname{LSD}_{\mathrm{f}}$ & 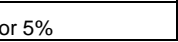 & 1.08 & 1.06 & 5.40 & 5.12 & 100.37 & 95.32 & 167.29 & 158.87 \\
\hline \multicolumn{10}{|c|}{$\mathrm{A} \times \mathrm{B}$ :Interaction } \\
\hline \multirow{5}{*}{ S.R } & Control & 9.07 & 9.01 & 447.0 & 448.2 & 2.846 & 2.836 & 8.357 & 8.394 \\
\hline & $\mathrm{Ca}+\mathrm{B}$ & 9.99 & 9.34 & 466.7 & 473.5 & 3.180 & 3.307 & 8.967 & 9.178 \\
\hline & $\mathrm{Y}+\mathrm{K}$ & 10.39 & 9.55 & 495.6 & 499.6 & 3.717 & 3.792 & 9.862 & 9.986 \\
\hline & $\mathrm{Y}+\mathrm{NAA}+\mathrm{K}$ & 11.07 & 9.94 & 526.3 & 527.3 & 4.289 & 4.308 & 10.816 & 10.847 \\
\hline & $\mathrm{Zn}+\mathrm{Cu}+\mathrm{Mn}$ & 9.75 & 9.32 & 471.0 & 472.5 & 3.260 & 3.289 & 9.101 & 9.148 \\
\hline \multirow{5}{*}{ H.A } & Control & 13.20 & 11.90 & 659.1 & 667.7 & 6.759 & 6.919 & 14.933 & 15.198 \\
\hline & $\mathrm{Ca}+\mathrm{B}$ & 14.88 & 14.36 & 742.8 & 736.1 & 8.315 & 8.190 & 17.525 & 17.318 \\
\hline & $\mathrm{Y}+\mathrm{K}$ & 13.51 & 12.81 & 697.9 & 696.9 & 7.480 & 7.462 & 16.133 & 16.104 \\
\hline & $\mathrm{Y}+\mathrm{NAA}+\mathrm{K}$ & 15.65 & 15.24 & 773.5 & 774.9 & 8.886 & 8.913 & 18.478 & 18.522 \\
\hline & $\mathrm{Zn}+\mathrm{Cu}+\mathrm{Mn}$ & 10.05 & 8.96 & 488.8 & 490.2 & 3.591 & 3.617 & 9.652 & 9.695 \\
\hline \multirow{5}{*}{ Poly. } & Control & 11.46 & 10.61 & 555.8 & 556.2 & 4.838 & 4.845 & 11.730 & 11.742 \\
\hline & $\mathrm{Ca}+\mathrm{B}$ & 12.38 & 11.10 & 582.3 & 584.9 & 5.330 & 5.379 & 12.550 & 12.632 \\
\hline & $\mathrm{Y}+\mathrm{K}$ & 12.60 & 11.98 & 616.7 & 617.8 & 5.970 & 5.991 & 13.618 & 13.651 \\
\hline & $\mathrm{Y}+\mathrm{NAA}+\mathrm{K}$ & 11.89 & 11.71 & 633.3 & 635.8 & 6.280 & 6.326 & 14.133 & 14.210 \\
\hline & $\mathrm{Zn}+\mathrm{Cu}+\mathrm{Mn}$ & 8.95 & 9.10 & 483.9 & 484.0 & 3.500 & 3.502 & 9.500 & 9.504 \\
\hline \multicolumn{2}{|c|}{ LSD for $5 \%$} & 1.87 & 1.83 & 9.34 & 8.88 & 173.85 & 165.10 & 289.75 & 275.15 \\
\hline
\end{tabular}

S.R: Straw rice costs H.A: humic acid poly: polymers Ca: calcium citrate

B: Borax Y: yeast NAA: naphthalene acetic acid

$\mathrm{K}$ : potassium citrate $\mathrm{Zn}+\mathrm{Cu}+\mathrm{Mn}$ : micronutrient 


\section{Fruit quality:-}

Data concerning fruit quality, i.e., TSS\%, Redus sugar\%, Non Redus sugar\%, Total sugar\%, T.carbohidrates\%, Acidity, \%Vitamin C , Carotenoid , Xanthophyll , and $\mathrm{NO}_{2}-\mathrm{N}$, as affected by soil, foliar application as well as their interactions during both seasons of 2012 and 2013 are present in Tables 6, 7 and 8.

Table (6): Effect of soil and foliar application as well as their interaction on TSS\%, R-sugar\%, N.R-sugar\%, Total sugar\% of pepper fruit during 2012 and 2013 seasons.

\begin{tabular}{|c|c|c|c|c|c|c|c|c|c|}
\hline \multirow{2}{*}{ Treat. } & \multirow[t]{2}{*}{ Char. } & \multicolumn{2}{|c|}{ T.SS\% } & \multicolumn{2}{|c|}{ R-sugar\% } & \multicolumn{2}{|c|}{ N.R-sugar\% } & \multicolumn{2}{|c|}{ Total sugar\% } \\
\hline & & $1^{\mathrm{st}}$ & $2^{\text {nd }}$ & $1^{\text {st }}$ & $2^{\text {nd }}$ & $1^{\text {st }}$ & $2^{\text {nd }}$ & $1^{\text {st }}$ & $2^{\text {nd }}$ \\
\hline \multicolumn{10}{|c|}{ A:Soil application } \\
\hline S.R & & 7.46 & 7.23 & 1.18 & 1.12 & 2.68 & 2.59 & 3.86 & 3.72 \\
\hline H.A & & 8.58 & 7.94 & 1.36 & 1.30 & 3.16 & 3.00 & 4.51 & 4.30 \\
\hline Poly. & & 7.19 & 6.94 & 1.17 & 1.12 & 2.62 & 2.53 & 3.80 & 3.62 \\
\hline $\mathrm{LSD}_{\text {at }}$ & & 0.09 & 0.06 & 0.03 & 0.04 & 0.04 & 0.05 & 0.03 & 0.06 \\
\hline \multicolumn{10}{|c|}{ B:Foliar application } \\
\hline \multicolumn{2}{|c|}{ Control } & 7.17 & 7.00 & 1.16 & 1.10 & 2.64 & 2.52 & 3.79 & 3.63 \\
\hline \multicolumn{2}{|c|}{$\mathrm{Ca}+\mathrm{B}$} & 7.68 & 7.48 & 1.22 & 1.17 & 2.80 & 2.70 & 4.02 & 3.87 \\
\hline \multicolumn{2}{|l|}{$\mathrm{Y}+\mathrm{K}$} & 7.78 & 7.53 & 1.26 & 1.20 & 2.88 & 2.76 & 4.14 & 3.96 \\
\hline \multicolumn{2}{|c|}{$\mathrm{Y}+\mathrm{NAA}+\mathrm{K}$} & 8.14 & 7.89 & 1.30 & 1.25 & 2.96 & 2.84 & 4.27 & 4.09 \\
\hline \multicolumn{2}{|c|}{$\mathrm{Zn}+\mathrm{Cu}+\mathrm{Mn}$} & 7.94 & 6.96 & 1.24 & 1.20 & 2.83 & 2.72 & 4.08 & 3.85 \\
\hline \multicolumn{2}{|c|}{ LSD at $5 \%$} & 0.08 & 0.06 & 0.04 & 0.03 & 0.03 & 0.03 & 0.05 & 0.04 \\
\hline \multicolumn{10}{|c|}{ AXB:Interaction } \\
\hline \multirow{5}{*}{ S.R } & Control & 6.69 & 6.46 & 1.12 & 1.08 & 2.48 & 2.39 & 3.60 & 3.46 \\
\hline & $\mathrm{Ca}+\mathrm{B}$ & 7.58 & 7.33 & 1.15 & 1.11 & 2.65 & 2.55 & 3.80 & 3.65 \\
\hline & $\mathrm{Y}+\mathrm{K}$ & 7.99 & 7.73 & 1.18 & 1.13 & 2.71 & 2.62 & 3.90 & 3.75 \\
\hline & $\mathrm{Y}+\mathrm{NAA}+\mathrm{K}$ & 8.09 & 7.84 & 1.23 & 1.17 & 2.81 & 2.73 & 4.03 & 3.89 \\
\hline & $\mathrm{Zn}+\mathrm{Cu}+\mathrm{Mn}$ & 6.93 & 6.79 & 1.20 & 1.14 & 2.77 & 2.68 & 3.98 & 3.82 \\
\hline \multirow{5}{*}{ H.A } & Control & 8.21 & 8.05 & 1.30 & 1.24 & 3.05 & 2.91 & 4.36 & 4.15 \\
\hline & $\mathrm{Ca}+\mathrm{B}$ & 8.49 & 8.29 & 1.37 & 1.32 & 3.18 & 3.07 & 4.55 & 4.39 \\
\hline & $\mathrm{Y}+\mathrm{K}$ & 8.03 & 7.77 & 1.38 & 1.34 & 3.21 & 3.09 & 4.59 & 4.43 \\
\hline & $\mathrm{Y}+\mathrm{NAA}+\mathrm{K}$ & 8.74 & 8.45 & 1.43 & 1.39 & 3.29 & 3.16 & 4.72 & 4.55 \\
\hline & $\mathrm{Zn}+\mathrm{Cu}+\mathrm{Mn}$ & 9.43 & 7.15 & 1.32 & 1.23 & 3.09 & 2.78 & 4.36 & 4.01 \\
\hline \multirow{5}{*}{ Poly. } & Control & 6.61 & 6.48 & 1.05 & 0.99 & 2.37 & 2.28 & 3.42 & 3.27 \\
\hline & $\mathrm{Ca}+\mathrm{B}$ & 6.97 & 6.83 & 1.15 & 1.09 & 2.58 & 2.48 & 3.73 & 3.56 \\
\hline & $\mathrm{Y}+\mathrm{K}$ & 7.32 & 7.11 & 1.21 & 1.14 & 2.71 & 2.56 & 3.93 & 3.70 \\
\hline & $\mathrm{Y}+\mathrm{NAA}+\mathrm{K}$ & 7.59 & 7.37 & 1.25 & 1.18 & 2.80 & 2.64 & 4.05 & 3.82 \\
\hline & $\mathrm{Zn}+\mathrm{Cu}+\mathrm{Mn}$ & 7.46 & 6.93 & 1.20 & 1.22 & 2.62 & 2.71 & 3.89 & 3.73 \\
\hline \multicolumn{2}{|c|}{ LSD at $5 \%$} & 0.12 & 0.10 & 0.06 & 0.06 & 0.06 & 0.05 & 0.08 & 0.07 \\
\hline
\end{tabular}

S.R: Straw rice costs H.A: humic acid poly: polymers Ca: calcium citrate

B: Borax Y: yeast NAA: naphthalene acetic acid

$\mathrm{K}$ : potassium citrate $\mathrm{Zn}+\mathrm{Cu}+\mathrm{Mn}$ : micronutrient

\section{Effect of soil application}

Concerning the effect of soil application on pepper plant fruit quality, the results reviewed in Table 6,7,8,9 and 10 show that the TSS\%, R-sugar\%, N.R-sugar\%, Total sugar\%, T.carbohidrates\%, Acidity, \%VIT C , Carotenoid ,Xanthophyll and $\mathrm{NO}_{2}-\mathrm{N}$ were significantly affected by using all soil 
application but the applications of humic acid at the rate $5 \mathrm{~kg} / \mathrm{fed}$. was found to be superior for increased TSS\%, R-sugar\%, N.R-sugar\%, Total sugar\% and chlorophyll a, b and total chlorophyllwith Abdel Fatah et al., (2008), Karakurt et al., (2009), and Selim et al., (2011) investigated the interactive effects of humic acid and water stress on chlorophyll and mineral nutrient contents of potato plants followed by adding straw rice costs at 4 ton/fed. and finally polymers.

Table (7): Effect of soil and foliar application as well as their interaction on T.carbohidrates\%, Acidity, \%VIT C of pepper fruit during 2012 and 2013 seasons.

\begin{tabular}{|c|c|c|c|c|c|c|c|}
\hline \multirow{2}{*}{ Treat } & \multicolumn{3}{|c|}{ Char.|T.carbohidrates $\%$} & \multicolumn{2}{|c|}{ Acidity\% } & \multicolumn{2}{|c|}{ V.C $(\mathrm{mg} / 100 \mathrm{~g})$} \\
\hline & & $1^{\mathrm{st}}$ & $2^{\text {nd }}$ & $1^{\text {st }}$ & $2^{\text {nd }}$ & $1^{\mathrm{st}}$ & $2^{\text {nd }}$ \\
\hline \multicolumn{8}{|c|}{ A:Soil application } \\
\hline \multicolumn{2}{|l|}{ S.R } & 13.35 & 14.55 & 0.67 & 0.67 & 91.91 & 91.04 \\
\hline \multicolumn{2}{|l|}{ H.A } & 14.66 & 15.99 & 0.84 & 0.83 & 98.65 & 97.57 \\
\hline \multicolumn{2}{|l|}{ Poly. } & 13.95 & 15.22 & 0.76 & 0.75 & 95.32 & 94.31 \\
\hline \multicolumn{2}{|c|}{ LSD at $5 \%$} & 0.04 & 0.04 & 0.04 & 0.04 & 0.26 & \\
\hline \multicolumn{8}{|c|}{ B:Foliar application } \\
\hline \multicolumn{2}{|c|}{ Control } & 13.61 & 14.92 & 0.71 & 0.69 & 95.70 & 92.88 \\
\hline \multicolumn{2}{|c|}{$\mathrm{Ca}+\mathrm{B}$} & 13.91 & 15.15 & 0.76 & 0.74 & 96.59 & 93.69 \\
\hline \multicolumn{2}{|c|}{$\mathrm{Y}+\mathrm{K}$} & 13.93 & 15.21 & 0.74 & 0.72 & 97.79 & 94.67 \\
\hline \multicolumn{2}{|c|}{$\mathrm{Y}+\mathrm{NAA}+\mathrm{K}$} & 14.17 & 15.54 & 0.78 & 0.76 & 98.91 & 95.83 \\
\hline \multicolumn{2}{|c|}{$\mathrm{Zn}+\mathrm{Cu}+\mathrm{Mn}$} & 14.31 & 15.45 & 0.79 & 0.83 & 87.48 & 94.48 \\
\hline \multicolumn{2}{|c|}{ LSD at $5 \%$} & 0.06 & 0.06 & 0.02 & 0.03 & 0.42 & 0.42 \\
\hline \multicolumn{8}{|c|}{ AXB:Interaction } \\
\hline \multirow{5}{*}{ S.R } & Control & 12.92 & 14.13 & 0.62 & 0.60 & 92.17 & 89.37 \\
\hline & $\mathrm{Ca}+\mathrm{B}$ & 13.07 & 14.27 & 0.64 & 0.64 & 92.63 & 89.87 \\
\hline & $\mathrm{Y}+\mathrm{K}$ & 13.33 & 14.54 & 0.65 & 0.64 & 93.73 & 90.90 \\
\hline & $\mathrm{Y}+\mathrm{NAA}+\mathrm{K}$ & 13.42 & 14.70 & 0.68 & 0.67 & 94.80 & 91.93 \\
\hline & $\mathrm{Zn}+\mathrm{Cu}+\mathrm{Mn}$ & 14.00 & 15.12 & 0.76 & 0.80 & 86.23 & 93.13 \\
\hline \multirow{5}{*}{ H.A } & Control & 14.29 & 15.68 & 0.80 & 0.79 & 98.87 & 96.07 \\
\hline & $\mathrm{Ca}+\mathrm{B}$ & 14.84 & 16.11 & 0.88 & 0.87 & 100.20 & 97.40 \\
\hline & $\mathrm{Y}+\mathrm{K}$ & 14.56 & 15.87 & 0.79 & 0.78 & 101.87 & 98.53 \\
\hline & $\mathrm{Y}+\mathrm{NAA}+\mathrm{K}$ & 15.00 & 16.49 & 0.87 & 0.86 & 103.70 & 100.17 \\
\hline & $\mathrm{Zn}+\mathrm{Cu}+\mathrm{Mn}$ & 14.62 & 15.79 & 0.82 & 0.86 & 88.61 & 95.70 \\
\hline \multirow{5}{*}{ Poly. } & Control & 13.63 & 14.95 & 0.71 & 0.68 & 96.07 & 93.20 \\
\hline & $\mathrm{Ca}+\mathrm{B}$ & 13.81 & 15.07 & 0.77 & 0.72 & 96.93 & 93.80 \\
\hline & $\mathrm{Y}+\mathrm{K}$ & 13.91 & 15.20 & 0.76 & 0.76 & 97.77 & 94.57 \\
\hline & $\mathrm{Y}+\mathrm{NAA}+\mathrm{K}$ & 14.08 & 15.42 & 0.77 & 0.76 & 98.23 & 95.40 \\
\hline & $\mathrm{Zn}+\mathrm{Cu}+\mathrm{Mn}$ & 14.31 & 15.45 & 0.78 & 0.82 & 87.59 & 94.60 \\
\hline \multicolumn{2}{|c|}{ LSD at $5 \%$} & 0.10 & 0.10 & 0.04 & 0.04 & 0.73 & 0.73 \\
\hline
\end{tabular}

S.R: Straw rice costs H.A: humic acid poly: polymers Ca: calcium citrate

B: Borax Y: yeast NAA: naphthalene acetic acid

$\mathrm{K}$ : potassium citrate $\mathrm{Zn}+\mathrm{Cu}+\mathrm{Mn}$ : micronutrient

\section{Effect of foliar application}

With regard to the effect of foliar application of different regulars on fruit quality, data in Table 6,7 and 8 clearly show that all previous parameters were increased with all foliar application. On the other hand, foliar application were significant TSS\%, R-sugar\%, N.R-sugar\%, Total sugar\%, 
T.carbohidrates\%, Acidity, \%VIT C, Carotenoid ,Xanthophyll and $\mathrm{NO}_{2}-\mathrm{N}$ during the both seasons. The highest values of TSS\%, R-sugar\%, N.Rsugar\%, Total sugar\% Carotenoid , Xanthophyll and $\mathrm{NO}_{2}-\mathrm{N}$ with using $\mathrm{Y}+\mathrm{NAA}+\mathrm{K}$ followed by using micronutrient solution $\mathrm{Zn}+\mathrm{Cu}+\mathrm{Mn}$, while spraying $\mathrm{Zn}+\mathrm{Cu}+\mathrm{Mn}$ followed by $\mathrm{Y}+\mathrm{NAA}+\mathrm{K}$ recorded the highest values of T.carbohidrates\%, Acidity, \%Vit C. Data illustrated in Table 9 and 10, show that there was a significant increase in chlorophyll a, b, total chlorophyll $(\mathrm{mg} / 100 \mathrm{~g})$ in pepper plant leaves and fruits in the two seasons as affected by foliar with different application. Plants sprayed with $\mathrm{Y}+\mathrm{NAA}+\mathrm{K}$ recorded the highest values of parameters under investigation compared with other treatments.

Table (8): Effect of soil and foliar application as well as their interaction on Carotenoid ,Xanthophyll and $\mathrm{NO}_{2}-\mathrm{N}$ of pepper fruit during 2012 and 2013 seasons.

\begin{tabular}{|c|c|c|c|c|c|c|}
\hline \multirow{2}{*}{$\begin{array}{l}\text { Char. } \\
\text { Treat. }\end{array}$} & \multicolumn{2}{|c|}{$\begin{array}{l}\text { Carotenoid } \\
(\mathrm{mg} / 100 \mathrm{~g})\end{array}$} & \multicolumn{2}{|c|}{$\begin{array}{c}\text { Xanthophyll } \\
\text { (mg/100g) }\end{array}$} & \multicolumn{2}{|c|}{$\mathrm{NO}_{2}-\mathrm{N}(\mathrm{mg} \backslash \mathrm{kg})$} \\
\hline & $1^{\text {st }}$ & $2^{\text {nd }}$ & $1^{\text {st }}$ & $2^{\text {nd }}$ & $1^{\text {st }}$ & $2^{\text {nd }}$ \\
\hline \multicolumn{7}{|c|}{ A:Soil application } \\
\hline S.R & 1.79 & 1.71 & 3.37 & 3.25 & 0.63 & 0.69 \\
\hline H.A & 2.62 & 2.49 & 4.82 & 4.59 & 0.81 & 0.89 \\
\hline Poly. & 2.21 & 2.13 & 4.18 & 3.99 & 0.67 & 0.73 \\
\hline LSD at $5 \%$ & 0.04 & 0.06 & 0.08 & 0.06 & 0.02 & 0.02 \\
\hline \multicolumn{7}{|c|}{ B:Foliar application } \\
\hline Control & 2.03 & 1.95 & 3.79 & 3.69 & 0.64 & 0.73 \\
\hline $\mathrm{Ca}+\mathrm{B}$ & 2.19 & 2.11 & 4.08 & 3.92 & 0.70 & 0.77 \\
\hline $\mathrm{Y}+\mathrm{K}$ & 2.23 & 2.14 & 4.12 & 3.98 & 0.73 & 0.77 \\
\hline $\mathrm{Y}+\mathrm{NAA}+\mathrm{K}$ & 2.35 & 2.27 & 4.40 & 4.24 & 0.78 & 0.86 \\
\hline $\mathrm{Zn}+\mathrm{Cu}+\mathrm{Mn}$ & 2.25 & 2.10 & 4.22 & 3.88 & 0.67 & 0.71 \\
\hline LSD at $5 \%$ & 0.05 & 0.04 & 0.04 & 0.04 & 0.03 & 0.03 \\
\hline \multicolumn{7}{|c|}{ AXB:Interaction } \\
\hline Control & 1.68 & 1.59 & 3.14 & 3.10 & 0.53 & 0.62 \\
\hline $\mathrm{Ca}+\mathrm{B}$ & 1.74 & 1.66 & 3.26 & 3.15 & 0.61 & 0.69 \\
\hline $\mathrm{Y}+\mathrm{K}$ & 1.79 & 1.72 & 3.38 & 3.27 & 0.65 & 0.65 \\
\hline $\mathrm{Y}+\mathrm{NAA}+\mathrm{K}$ & 1.93 & 1.85 & 3.63 & 3.49 & 0.73 & 0.79 \\
\hline $\mathrm{Zn}+\mathrm{Cu}+\mathrm{Mn}$ & 1.83 & 1.73 & 3.42 & 3.26 & 0.65 & 0.69 \\
\hline Control & 2.43 & 2.34 & 4.44 & 4.30 & 0.78 & 0.89 \\
\hline $\mathrm{Ca}+\mathrm{B}$ & 2.71 & 2.60 & 5.01 & 4.77 & 0.86 & 0.93 \\
\hline $\mathrm{Y}+\mathrm{K}$ & 2.57 & 2.48 & 4.74 & 4.57 & 0.82 & 0.91 \\
\hline $\mathrm{Y}+\mathrm{NAA}+\mathrm{K}$ & 2.74 & 2.66 & 5.07 & 4.92 & 0.86 & 0.96 \\
\hline $\mathrm{Zn}+\mathrm{Cu}+\mathrm{Mn}$ & 2.68 & 2.37 & 4.85 & 4.39 & 0.70 & 0.74 \\
\hline \multirow{6}{*}{\begin{tabular}{|l|} 
Control \\
$\mathrm{Ca}+\mathrm{B}$ \\
$\mathrm{Y}+\mathrm{K}$ \\
$\mathrm{Y}+\mathrm{NAA}+\mathrm{K}$ \\
$\mathrm{Zn+Cu+Mn}$ \\
\end{tabular}} & 1.97 & 1.91 & 3.80 & 3.69 & 0.61 & 0.67 \\
\hline & 2.13 & 2.06 & 3.97 & 3.84 & 0.65 & 0.71 \\
\hline & 2.31 & 2.22 & 4.25 & 4.09 & 0.71 & 0.75 \\
\hline & 2.38 & 2.29 & 4.50 & 4.33 & 0.74 & 0.83 \\
\hline & 2.25 & 2.18 & 4.37 & 3.98 & 0.65 & 0.69 \\
\hline & 0.09 & 0.07 & 0.07 & 0.08 & 0.05 & 0.06 \\
\hline $\begin{array}{l}\text { S.R: Straw rice costs } \\
\text { B: Borax Y: yeast }\end{array}$ & $\begin{array}{l}\text { H.A: hu } \\
\text { NAA: } n\end{array}$ & $\begin{array}{l}\text { acid } \\
\text { halene }\end{array}$ & $\begin{array}{l}\text { y: polyn } \\
\text { tic acid }\end{array}$ & & Ium c & \\
\hline
\end{tabular}

$\mathrm{K}$ : potassium citrate $\mathrm{Zn}+\mathrm{Cu}+\mathrm{Mn}$ : micronutrient 
These findings are also in agreement with the results of Wanas (2006), Mady (2009), and Olaiya (2010b) reported that the increase in photosynthetic pigments formation could be attributed to the role of yeast cytokinins in delaying the aging of leaves by reducing the degradation of chlorophyll and enhancing the protein and RNA synthesis. As for NAA Sridhar et al., (2009) and Abou El-Yazied and Mady (2011) found that the two concentrations of each applied yeast extract or naphthalene acetic acid obviously increased photosynthetic pigments. The effect of potassium Lin Duo and H. Danfeng (2003), Sarrwy et al., (2010) and El-Bassiony et al., (2012) stated that the highest potassium fertilization rate $(200 \mathrm{~kg} / \mathrm{fed}$.) gave the highest values of total chlorophyll

\section{Effect of interaction between soil and foliar application:}

The interaction of soil and foliar application significantly enhances fruit quality as compared with the untreated plants (sprayed with water) in Table 6, 7 and 8 , it is clear that spraying either $\mathrm{Y}+\mathrm{NAA}+\mathrm{K}$ and $\mathrm{Zn}+\mathrm{Cu}+\mathrm{Mn}$ pushed to increase the previous parameters under adding humic acid at rate of $5 \mathrm{~kg} / \mathrm{fed}$. during both seasons.

The positive effects of the humic acid was observed on the studies such as Abdel Fatah et al., (2008), Karakurt et al., (2009) and Kazemi (2013) resulted that a combination of humic acid+ potassium nitrate (40 ppm $+100 \mathrm{mg} / \mathrm{L} \mathrm{K}$ ) was the most effective in increasing fruit quality and total soluble solid content of the fruit.

The enhancing effect of yeast application might be due to that yeast cytokinins enhance the accumulation of soluble metabolites, (Shalaby and ElNady, 2008). On the other hand, Kataoka et al., (2009) found that the addition of auxin solutions for fruit setting at anthesis increased the amount of sugar content per fruit at maturity in tomato cultivar. Also, This superiority might be due to that amino green compound contains many amino acids as well as some growth regulators and vitamins which stimulate and enhance the metabolism processes in plant tissues. Whereas, the previous studies have proved that, amino acids, can directly or indirectly influenc the physiological activities of the plants (Shaheen et al., 2010). Similar result as effect of yeast recorded with Wanas (2006); Hussain and Khalaf (2007), and Ghoname et al., (2010) showed that yeast solutions 1,2 and $3 \mathrm{~g} / \mathrm{l}$ improved fruit quality in terms of total Soluble Solids (TSS), total Acidity and ascorbic acid contents of sweet pepper showed also similar positive responses compared to untreated ones.

As for NAA, Sridhar et al., (2009) studied the effect of foliar spray of naphthalene acetic acid [NAA] (50, 100 and 150 ppm) on bell pepper. All treatments significantly increased total chlorophyll, ascorbic acid and nitrate reductase activity were also increased.

In plants, the potassium is related to the synthesis of proteins and carbohydrates, sugars and starch storage and this stimulated the growth and improved utilization of water and the resistance to pests and diseases Faquin, (1994). Balibrea et al., (2006) have reported that an increase of TSS in tomato fruits may depend on a higher sugar import and accumulation. Sofia, (2008) mentioned that the increase of TSS together, reducing sugars in the fruits of plants grown in addition with the higher $\mathrm{K}$ levels 
in the nutrient solution confirm that $\mathrm{K}$ played an important role in the configuration of quality profile in tomato fruits.

It could be concluded that spraying with Yeast $(10 \mathrm{mg} / \mathrm{l})+\mathrm{NAA}$ (naphthalene acetic acid $2.5 \mathrm{mg} / \mathrm{l})+\mathrm{K}$ (citrate potassium $4 \mathrm{mg} / \mathrm{l}$ ) combined with $5 \mathrm{~kg} / \mathrm{fed}$. humic acid increased the yield and quality of pepper plant. With respect to the effect of interaction between soil, and foliar application on chemical composition of pepper plant leaves and fruits, it is evident from data present in Tables 9 and 10 that the mean values of chlorophyll a, b, and total chlorophyll $(\mathrm{mg} / 100 \mathrm{~g})$ were significantly affected and the best records of previous characters were obtained by plants fertilized with humic acid (5 $\mathrm{kg} / \mathrm{fed}$ ) and sprayed with $\mathrm{Y}+\mathrm{NAA}+\mathrm{K}$ compared with the other treatments and the control which recorded the lowest values of the parameters during the both seasons of the experiments.

Table (9): Effect of soil and foliar application as well as their interaction on chlorophyll a, b, and total chlorophyll $(\mathrm{mg} / \mathbf{1 0 0 \mathrm { g }})$ of pepper leaves during 2012 and 2013 seasons.

\begin{tabular}{|c|c|c|c|c|c|c|c|}
\hline \multirow{2}{*}{ Treat. } & \multirow[t]{2}{*}{ Char. } & \multicolumn{2}{|c|}{$\begin{array}{c}\text { Chlorophyll a } \\
(\mathrm{mg} / 100 \mathrm{~g})\end{array}$} & \multicolumn{2}{|c|}{$\begin{array}{c}\text { Chlorophyll b } \\
\text { (mg/100g) }\end{array}$} & \multicolumn{2}{|c|}{$\begin{array}{c}\text { Total } \\
\text { chlorophyll } \\
\text { (mg/100g) }\end{array}$} \\
\hline & & $1^{\mathrm{st}}$ & $2^{\text {nd }}$ & $1^{\text {st }}$ & $2^{\text {nd }}$ & $1^{\text {st }}$ & $2^{\text {nd }}$ \\
\hline \multicolumn{8}{|c|}{ A: Soil application } \\
\hline S.R & & 0.961 & 0.914 & 0.476 & 0.553 & 1.470 & 1.467 \\
\hline H.A & & 0.989 & 0.949 & 0.498 & 0.568 & 1.585 & 1.518 \\
\hline Poly. & & 0.932 & 0.892 & 0.459 & 0.533 & 1.433 & 1.425 \\
\hline LSD at $5 \%$ & & 0.010 & 0.005 & 0.476 & 0.004 & 0.004 & 0.008 \\
\hline \multicolumn{8}{|c|}{ B: Foliar application } \\
\hline Control & & 0.949 & 0.908 & 0.470 & 0.536 & 1.420 & 1.444 \\
\hline $\mathrm{Ca}+\mathrm{B}$ & & 0.961 & 0.926 & 0.479 & 0.553 & 1.440 & 1.479 \\
\hline $\mathrm{Y}+\mathrm{K}$ & & 0.964 & 0.928 & 0.479 & 0.551 & 1.443 & 1.479 \\
\hline $\mathrm{Y}+\mathrm{NAA}+\mathrm{K}$ & & 0.976 & 0.937 & 0.485 & 0.562 & 1.461 & 1.499 \\
\hline $\mathrm{Zn}+\mathrm{Cu}+\mathrm{Mn}$ & & 0.952 & 0.893 & 0.475 & 0.555 & 1.716 & 1.448 \\
\hline LSD at $5 \%$ & & 0.008 & 0.007 & 0.004 & 0.005 & 0.004 & 0.008 \\
\hline \multicolumn{8}{|c|}{ AXB: Interaction } \\
\hline \multirow{5}{*}{ S.R } & Control & 0.947 & 0.905 & 0.471 & 0.542 & 1.418 & 1.447 \\
\hline & $\mathrm{Ca}+\mathrm{B}$ & 0.957 & 0.925 & 0.475 & 0.555 & 1.431 & 1.480 \\
\hline & $\mathrm{Y}+\mathrm{K}$ & 0.964 & 0.926 & 0.478 & 0.554 & 1.442 & 1.480 \\
\hline & $\mathrm{Y}+\mathrm{NAA}+\mathrm{K}$ & 0.972 & 0.933 & 0.482 & 0.560 & 1.454 & 1.493 \\
\hline & $\mathrm{Zn}+\mathrm{Cu}+\mathrm{Mn}$ & 0.963 & 0.880 & 0.478 & 0.552 & 1.604 & 1.432 \\
\hline \multirow{5}{*}{ H.A } & Control & 0.981 & 0.939 & 0.487 & 0.553 & 1.468 & 1.492 \\
\hline & $\mathrm{Ca}+\mathrm{B}$ & 0.996 & 0.959 & 0.502 & 0.575 & 1.497 & 1.534 \\
\hline & $\mathrm{Y}+\mathrm{K}$ & 0.992 & 0.957 & 0.497 & 0.568 & 1.489 & 1.524 \\
\hline & $\mathrm{Y}+\mathrm{NAA}+\mathrm{K}$ & 1.015 & 0.980 & 0.508 & 0.585 & 1.523 & 1.565 \\
\hline & $\mathrm{Zn}+\mathrm{Cu}+\mathrm{Mn}$ & 0.960 & 0.912 & 0.495 & 0.561 & 1.947 & 1.473 \\
\hline \multirow{5}{*}{ Poly. } & Control & 0.920 & 0.880 & 0.453 & 0.512 & 1.373 & 1.391 \\
\hline & $\mathrm{Ca}+\mathrm{B}$ & 0.931 & 0.893 & 0.460 & 0.529 & 1.391 & 1.422 \\
\hline & $\mathrm{Y}+\mathrm{K}$ & 0.936 & 0.902 & 0.463 & 0.531 & 1.399 & 1.433 \\
\hline & $\mathrm{Y}+\mathrm{NAA}+\mathrm{K}$ & 0.941 & 0.898 & 0.464 & 0.540 & 1.406 & 1.438 \\
\hline & $\mathrm{Zn}+\mathrm{Cu}+\mathrm{Mn}$ & 0.933 & 0.887 & 0.454 & 0.552 & 1.597 & 1.439 \\
\hline LSD at $5 \%$ & & 0.014 & 0.011 & 0.008 & 0.008 & 0.008 & 0.013 \\
\hline
\end{tabular}


Table (10): Effect of soil and foliar application as well as their interaction on chlorophyll $\mathrm{a}, \mathrm{b}$, and total chlorophyll $(\mathrm{mg} / \mathbf{1 0 0 \mathrm { g }})$ of pepper fruits during 2012 and 2013 seasons.

\begin{tabular}{|c|c|c|c|c|c|c|c|}
\hline \multirow[t]{2}{*}{ Treat. } & \multirow[t]{2}{*}{ Char. } & \multicolumn{2}{|c|}{$\begin{array}{c}\text { Chlorophyll a } \\
\text { (mg/100g) }\end{array}$} & \multicolumn{2}{|c|}{$\begin{array}{l}\text { Chlorophyll b } \\
\text { (mg/100g) }\end{array}$} & \multicolumn{2}{|c|}{$\begin{array}{c}\text { Total } \\
\text { chlorophyll } \\
(\mathrm{mg} / 100 \mathrm{~g})\end{array}$} \\
\hline & & $1^{\mathrm{st}}$ & $2^{\text {nd }}$ & $1^{\text {st }}$ & $2^{\text {nd }}$ & $1^{\text {st }}$ & $2^{\text {nd }}$ \\
\hline \multicolumn{8}{|c|}{ A: Soil application } \\
\hline S.R & & 0.612 & 0.599 & 0.435 & 0.417 & 1.046 & 1.017 \\
\hline H.A & & 0.641 & 0.628 & 0.452 & 0.436 & 1.093 & 1.063 \\
\hline Poly. & & 0.602 & 0.590 & 0.435 & 0.417 & 1.037 & 1.007 \\
\hline LSD a & & 0.012 & 0.011 & 0.009 & 0.004 & 0.014 & 0.010 \\
\hline \multicolumn{8}{|c|}{ B: Foliar application } \\
\hline Contr & & 0.613 & 0.600 & 0.428 & 0.410 & 1.041 & 1.010 \\
\hline $\mathrm{Ca}+\mathrm{B}$ & & 0.615 & 0.603 & 0.438 & 0.425 & 1.053 & 1.028 \\
\hline $\mathrm{Y}+\mathrm{K}$ & & 0.617 & 0.604 & 0.446 & 0.427 & 1.063 & 1.032 \\
\hline $\mathrm{Y}+\mathrm{NA}$ & & 0.629 & 0.616 & 0.451 & 0.430 & 1.080 & 1.047 \\
\hline $\mathrm{Zn}+\mathrm{C}$ & & 0.616 & 0.604 & 0.441 & 0.424 & 1.058 & 1.028 \\
\hline LSDat & & 0.010 & 0.010 & 0.006 & 0.003 & 0.010 & 0.009 \\
\hline \multicolumn{8}{|c|}{ AXB: Interaction } \\
\hline \multirow{5}{*}{ S.R } & Control & 0.602 & 0.590 & 0.420 & 0.404 & 1.022 & 0.993 \\
\hline & $\mathrm{Ca}+\mathrm{B}$ & 0.615 & 0.603 & 0.439 & 0.422 & 1.054 & 1.025 \\
\hline & $\mathrm{Y}+\mathrm{K}$ & 0.608 & 0.596 & 0.444 & 0.428 & 1.052 & 1.024 \\
\hline & $\mathrm{Y}+\mathrm{NAA}+\mathrm{K}$ & 0.625 & 0.612 & 0.448 & 0.427 & 1.073 & 1.039 \\
\hline & $\mathrm{Zn}+\mathrm{Cu}+\mathrm{Mn}$ & 0.609 & 0.597 & 0.421 & 0.405 & 1.030 & 1.002 \\
\hline \multirow{5}{*}{ H.A } & Control & 0.654 & 0.640 & 0.447 & 0.429 & 1.101 & 1.070 \\
\hline & $\mathrm{Ca}+\mathrm{B}$ & 0.638 & 0.625 & 0.445 & 0.436 & 1.083 & 1.060 \\
\hline & $\mathrm{Y}+\mathrm{K}$ & 0.636 & 0.623 & 0.455 & 0.437 & 1.091 & 1.060 \\
\hline & $\mathrm{Y}+\mathrm{NAA}+\mathrm{K}$ & 0.648 & 0.635 & 0.461 & 0.442 & 1.109 & 1.077 \\
\hline & $\mathrm{Zn}+\mathrm{Cu}+\mathrm{Mn}$ & 0.627 & 0.614 & 0.454 & 0.436 & 1.081 & 1.050 \\
\hline \multirow{5}{*}{ Poly. } & Control & 0.583 & 0.571 & 0.416 & 0.396 & 0.999 & 0.967 \\
\hline & $\mathrm{Ca}+\mathrm{B}$ & 0.593 & 0.581 & 0.429 & 0.419 & 1.022 & 1.000 \\
\hline & $\mathrm{Y}+\mathrm{K}$ & 0.606 & 0.594 & 0.439 & 0.417 & 1.045 & 1.011 \\
\hline & $\mathrm{Y}+\mathrm{NAA}+\mathrm{K}$ & 0.614 & 0.602 & 0.444 & 0.422 & 1.057 & 1.024 \\
\hline & $\mathrm{Zn}+\mathrm{Cu}+\mathrm{Mn}$ & 0.613 & 0.601 & 0.449 & 0.431 & 1.062 & 1.032 \\
\hline \multicolumn{2}{|c|}{ LSD at $5 \%$} & 0.017 & 0.017 & 0.011 & 0.005 & 0.017 & 0.016 \\
\hline
\end{tabular}

S.R: Straw rice costs H.A: humic acid poly: polymers Ca: calcium citrate

B: Borax Y: yeast NAA: naphthalene acetic acid

$\mathrm{K}$ : potassium citrate $\mathrm{Zn}+\mathrm{Cu}+\mathrm{Mn}$ : micronutrient

\section{CONCLUSION}

These all findings emphasize the importance of determining the interactive effects between soil application and foliar spray from different enhance materials to find out the optimum combinations for maximum early, total yield and special fruit quality. The treatment of adding $5 \mathrm{~kg} / \mathrm{fed}$. humic acid with spray $\mathrm{Y}+\mathrm{NAA}+\mathrm{K}$ followed by the spray treatment of $\mathrm{Ca}+\mathrm{B}$ under the same soil application considered the best combination and it is recommended for pepper plant grown under similar field conditions in order to get higher economical yield. 


\section{REFERENCES}

A.O.A.C. (2000) "Official methods of Analysis" Twelfth Ed. Published by the Association of Official Analytical chemists, Benjamin, France line station, Washington.Dc.

Abdel Fatah, G. H.; A. Boshra, S. M. Shahin (2008). The role of humic acid in reducing the harmful effect of irrigation with saline water on tifway turf. J. Bio. Chem. Environ. Sci., 3(1): 75-89.

Abou El-Yazied, A. and M. A. Mady (2011). Effect of naphthalene acetic acid and yeast extract on growth and productivity of tomato (lycopersicon esculentum mill.) plants. Res. J. Agric. \& Biol. Sci., 7(2): 271-281.

Alam, S.M. and M.A. Khan (2002). Fruit yield of tomato as affected by NAA spray. Asian Journal of Plant Sciences, 1(1): 11-24.

Anonymous, (2010). Humic and fulvic acids: The black gold of agriculture. http://www.humintech.com/pdf/humicfulvicacids.pdf (Access date: 10.08.2010).

Arancon, N.Q.; C.A. Edwards, S. Lee and R. Byrne (2006). Effects of humic acids from vermicomposts on plant growth. European J. Soil Bio., 42(Suppl.1): S65-S69.

Balibrea, M. A.; C. Martínez-Andújar, J. Cuartero, M. C. Bolarín, and F. Pérez-Alfocea (2006). The high fruit soluble sugar content in wild Lycopersicon species and their hybrids with cultivars depends on sucrose import during ripening rather than on sucrose metabolism. Funct. Plant Biol., 33: 279-288.

Bevilacqua, A.; M. R. Corbo, M. Mastromatteo and M. Sinigaglia (2008). Combined effects of $\mathrm{pH}$, yeast extract, carbohydrates and diammonium hydrogen citrate on the biomass production and acidifying ability of a probiotic Lactobacillus plantarum strain, isolated from table olives, in a batch system. World J. Microbiol Biotechnol., 24: 17211729.

Bhuvaneswari, G.; R. Sivaranjani, S. Reeth and K. Ramakrishnan (2013). Application of Nitrogen and Potassium efficiency on the growth and yield of chilli Capsicum annuum L. Int. J. Curr. Microbiol. App. Sci., 2(12): 329-337.

Boatright, J. L.; D. E. Balint, W. A. Mackay, and J. M. Zajicek (1997). Incorporation of a hydrophilic polymer into annual landscape beds, J. Environ. Hort. 15, 37-40

Bremner, J. M. and C. S. Mulvany (1982). Nitrogen total P. 595. 616. in Page, A. L. et al., (ed.) "Methods of Soil Analysis". Part2: Chemical and Microbiological Properties. Amer. Soc. of Agron., Inc., Madison, Wis., USA.

Dixon,J.B and S.B.Weed(1989).Page 95 In"Minerals in soil Environments".Soil Science Socity of America, Madison, Wisconsin, 1244 pages

Dubois, M., A. Gilles, J. K. Homilton, P. A. Rebers and P. A. Smith (1956) A colorimetric method for determination of sugars and related substances.Anal. Chem., 28(3): 350-356. 
El-Bassiony, A. M.; Z. F. Fawzy; E. H. Abd El-Samad and G. S. Riad (2012). Growth, Yield and Fruit Quality of Sweet Pepper Plants (Capsicum annuum L.) as Affected by Potassium Fertilization. International Journal of Agr. \& Env. (04): 54-61.

El-Bassiony, A. M.; Z. F. Fawzy; E. H. Abd El-Samad and G. S. Riad (2012). Growth, yield and fruit quality of sweet pepper plants (Capsicum annuum L.) as affected by potassium fertilization. International Journal of Agr. \&Env. (04): 54-61.

El-Tohamy, W.A., H.M. El-Abagy and N.H.M. El-Greadly(2008). Studies on the effect of putrescine, yeast and vitamin $C$ on growth, yield and physiological responses of eggplant (Salanummelongena L.) under sandy soil conditions. Australian Journal of Basic and Applied. Science, 2(2): 296300.

Eyheraguibel, B.; J. Silvestre, and P. Morard (2008). Effects of humic substances derived from organic waste enhancement on the growth and mineral nutrition of maize, Bioresource Technology. 99: 4206-4212.

Faquin, V. (1994). Mineral nutrition of plants. Lavras: ESAL-FAEPE: p. 227.

Fathy, S.L. and S. Farid(1996). Effect of some chemical treatments, yeast preparation and royal jelly on some vegetable crops growing in late summer season to induce their ability towards better thermal tolerance. J. Agric. Sci. Mansoura Univ., 25(4): 2215-2249.

Fawzy, Z.F.; A.G. Behairy and S.A. Shehata(2005). Effect of potassium fertilizer ongrowth and yield of sweet pepper plants (Capsicum annuum, L.). Egypt. J. Agric. Res., 2(2): 599-610.

Ghoname, A. A., M. A. El-Nemr, A. M. R. Abdel-Mawgoud, and W. A. ElTohamy (2010). Enhancement of Sweet pepper crop growth and production by application of biological, organic and nutritional solutions. Research Journal of Agriculture and Biological Sciences, 6(3), 349-355.

Gomaa A.M., S.S. Moawad, I.M.A. Ebadah and H.A. Salim(2005). Application of bio-organic farming and its influence on certain pests infestation, growth and productivity of potato plants. Journal of Applied Sciences Research, 1(2): 205-211.

Gomez, K. A. and A. A. Gomez (1984)."Statistical Procedures for Agricultural Research". John Wiley and Sons, Inc., New York. pp: 680.

Goodwine, T. W. (1965). Quantitative analysis of the chloroplast pigments. Academic Press, London and New York.

Hussain, W. and L. Khalaf (2007). Effect of foliar spraying with yeast solution on growth and yield of potato plant cv. desiree. $\mathrm{html} / \mathrm{www}$.tropentage.de/2007/abstracts/links/khalaf. FPRAXY 90.

Jackson, M. L. (1967). "Soil Chemical Analysis Advanced Course" Puble. By the auther, Dept. of Soils, Univ. of Wise., Madison 6, Wishensin, USA

Karakurt, Y.; H. Unlu, H. Unluand H. Padem (2009). The influence of foliar and soil fertilization of humic acid on yield and quality of pepper.ActaAgriculturaeScandinavica, Section B - Soil \& Plant Sci., 59 (3): 233-237. 
Kataoka, K., Y. Yashiro, T. Habu, K. Sunamoto and A. Kitajima (2009). The addition of gibberellic acid to auxin solutions increases sugar accumulation and sink strength in developing auxin-induced parthenocarpic tomato fruits. Scientia Horticulturae, 123: 228-233.

Kazemi, M. (2013).Effect of foliar application of humic acid and potassium nitrate on cucumber growth.Bull. Env. Pharmacol. Life Sci., 2 (11): $03-$ 06.

Koller, H. R. (1972). Leaf area and leaf weight relationship in soyabean canopy. Crop Sci., 12: 180-183.

Lin, Duo and H. Danfeng (2003). Effects of potassium levels on photosynthesis and fruit quality of muskmelon in culture medium. Acta Horti. Sinica., 30(2): 221-223.

Mady, M. A. (2009). Effect of foliar application With yeast extract and zinc on fruit setting and yield of faba bean (vicia faba I). Agric. Botany Dept, Fac of Agric, Benha Univ J. Biol. Chem Environ. Sci., 4(2): 109-127.

Mahmoud, A. R., and M. M. Hafez, (2010). Increasing productivity of potato plants (Solanum tubersoum L.) by using potassium fertilizer and humic acid application. Int. J. Acad. Res. 2:83-88.

Majumdar, S.P.;R. L. Meena and G. D. S. Baghel(2000). Effect of levels of compaction and potassium on yield and quality of tomato and chilli crops grown on highly permeable soils. J. Indian Soc. Soil Sci., 48 (2) : 215-220.

Mikkelsen, R. L. (1999). Usinghydrophilic polymers to control nutrient release. Nutr. Cycl. Agroechosys. 38(1), 53-59.

Morsi, M.K.; B. El-Magoli, N.T. Saleh, E.M. El-Hadidy and H.A. Barakat(2008). Study of antioxidants and anticancer activity licorice Glycyrrhizaglabra extracts. Egyptian J. Nutr. And Feeds, 2(33): 177203.

Olaiya, C. O. (2010b). Presowing Bioregulator Seed Treatments Increase the Seedling Growth and Yield of Tomato (Lycopersicon esculentum). J. Plant Growth Regul., 29: 349-356.

Padem, H. and A. Ocal (1999). Effects of humic acid applications on yield and some characteristics of processing tomato. Acta Hort., 487: 159-164.

Peterburgski, A. V. (1968). "Hand Book of Agronomic Chemistry". Kolas publishing House, Moscow, (in Russian), pp. 29-86.

Peterson, D. (2002). Hydrophilic polymers and uses in landscape. Horticul. Sci., Vol 75.

Piper, C. S. (1950). "Soil and plant analysis". Inter Science Publishers Inc. New York.

Pregle, E. (1945). "Quantitative Organic Micro-Analysis" 4th Ed. J. Chudrial, London.

Sadasivam, S. and A. Manickam (1996).Biochemical Methods, $2^{\text {nd }}$ Ed.New Age international (P) Limitid Publishers, New Delhi P. 42-43. 
Sarhan, T. and O.K. Abdullah (2010). Effect of azotobacter inoculation, dry bread yeast suspension and varying levels of urea on growth of potato Cv. desiree.html/www.tropentage.de/2010/abstracts/full/628.

Sarrwy, S. M. A.; A. Enas, and H. S. A. Hassan (2010). Effect of foliar spray with potassium nitrate and mono-potassium phosphate on leaf mineral contents, fruit set, yield and fruit quality of Picual olive trees grown under sandy soil conditions. American-Eurasian J. Agric and Environ. Sci., 8(4): 420-430.

Selim, E. M.; S. I. Shedeed, F. F. Asaad, and A. S. El-Neklawy, (2011). Interactive effects of humic acid and water stress on chlorophyll and mineral nutrient contents of potato plants. J. Appl. Sci. Res., 7:531-537.

Shaheen, A. M.; A. R. Fatma, A. M. Hoda, A. E. Habib, and M. M. H. Baky (2010). Nitrogen soil dressing and foliar spraying by sugar and amino acids as affected the growth, yield and its quality of onion plant. Journal of American Science, 6(8), 420-427.

Shalaby, M. E. and M. F. El-Nady (2008). Application of Saccharomyces cerevisiae as a biocontrol agent against Fusarium infection of sugar beet plants. Acta Biologica Szegediensis, 52(2): 271-275.

Shehata, S. A.; Z. F. Fawzy and H. R. El-Ramady (2012). Response of cucumber plants to foliar application of chitosan and yeast under greenhouse conditions. Aust. J. Basic \& Appl. Sci., 6(4): 63-71.

Singh, J. P., (1988). A rapid method for fertermination of nitrate in soil and plant extracts. Plant and soil. 110: 137-139.

Sofia, C. (2008). Influence of Potassium and Genotype on Vitamin E Content and Reducing Sugar of Tomato Fruits. Hortscience., 43(7): 2048-2051.

Sridhar, G.; R.V. Koti, M.B. Chetti and S.M. Hiremath(2009). Effect of naphthalene acetic acid and mepiquat chloride on physiological components of yield in bell pepper (capsicum annuum I.). J. Agric. Res., 47(1): 53-62.

U S Salinity Laboratory Staff (1954). Diagnosis and Improvement of Saline and Alkali Soils. USDA Agric. Hand Book No. 60, Washington, D.C.

Wanas, A. L. (2006). Trails for improving growth and productivity of tomato plants grown in winter. Annals. Agric. Sci. Moshtohor., 44(3): 214-231. 
تأثير بعض المعاملات الارضيه ومعاملات الرش على الإتتاجية والجودة لمحصول

القلقل

(1) النمو الخضرى والمحصول و التركيب الكيماوي .

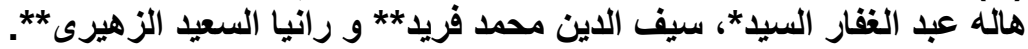

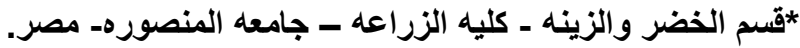

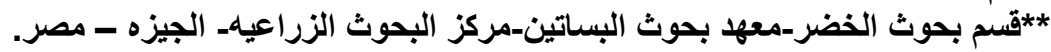

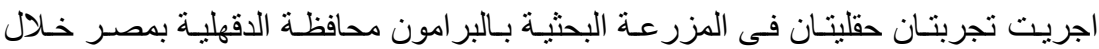

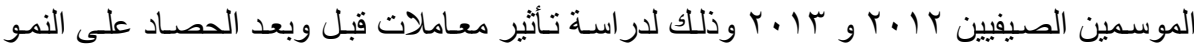
والانتاجيه لثمار نبات الفلفل صنف كاليفورنيا.

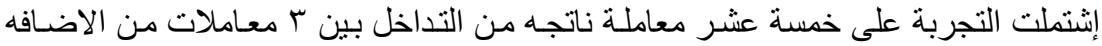

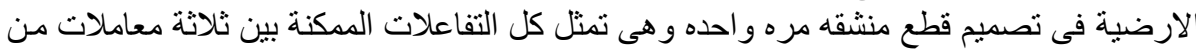

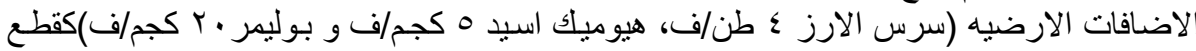

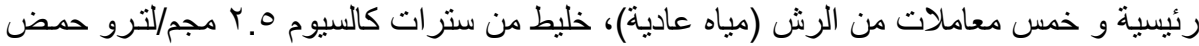

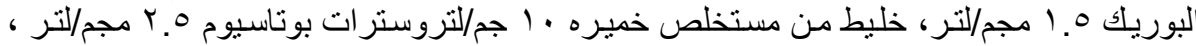

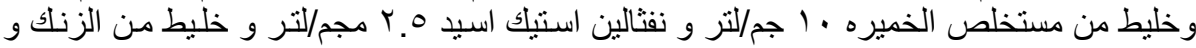
النحاس و المنجنيز فى صوره كبريتيه) كقطع فر عبة وز عت المعاملات فى ثلاثة مكررات . وقا اوضحت النتائج الاتي:-

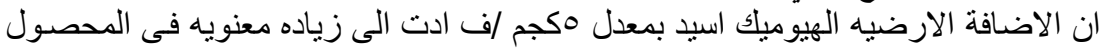

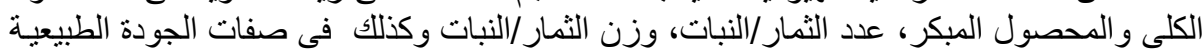

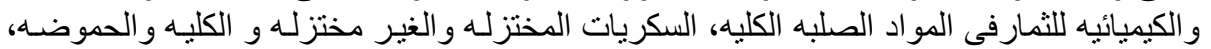

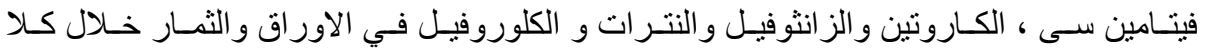

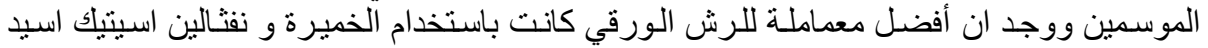

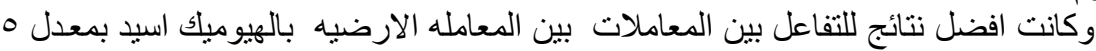

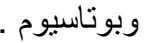
كجم/للفدان والرش بالخميرة ونفظالين اسيتيكاك اسيد وبوتاسيوم . 